\title{
Performance Evaluation of LPG-PCA Algorithm in Deblurring of CT and MRI Images
}

\author{
R.Hari Kumar \\ Professor \\ Bannari Amman Institute of \\ Technology \\ Sathyamangalam,Erode, India.
}

\author{
B.Vinoth Kumar, PhD. \\ Assistant Prof Sr.Grade \\ Bannari Amman Institute of \\ Technology \\ Sathyamangalam,Erode, India.
}

\author{
S.Gowthami \\ PG Scholar \\ Bannari Amman Institute of \\ Technology \\ Sathyamangalam,Erode, India.
}

\begin{abstract}
This paper presents the performance analysis of the LPG-PCA algorithm in deblurring of medical images. Medical images containing lot of information which are often affected by noise and artifacts, which leads to the inefficient diagnosis. LPG-PCA which is a statistical decorrelation technique is found to be one of the efficient methods which could be used in improving the performance of medical images. For better preservation of fine structures in an image, a pixel and its nearest neighbors are modeled as a vector variable whose training samples are selected using a moving window in the image. Such a local vector variable preservation leads to the selection of the similar intensity characteristics. This property of LPG-PCA technique is applied in image deblurring process using adaptive sparse domain regularization technique. This method involves clustering of data and finding the Sub dictionary of each cluster using LPG-PCA. Then the dictionary for input patch is selected using SVD technique and deblurring is done using regularization. Performance analysis of this technique is found using various image quality measures and results are found to be efficient than other conventional methods.
\end{abstract}

\section{Keywords}

Principle Component Analysis, Local Pixel Grouping, Deblurring, Image Quality Measures.

\section{INTRODUCTION}

Noise will be inevitably introduced during the image acquisition process of medical images. These images are often affected by random noise produced during the image acquisition process. Presence of noise not only reduces the visual quality of the images but also lowers the visibility of low contrast objects even it's of fine importance [1]. Denoising is done in the noisy images in order to recover the original image with essential characteristics. After applying an effective denoising scheme the original image will be recovered from the noisy environment even though some edge information's are lost due to denoising [2]. But edge information is one of the basic things for diagnosis in medical images. In order to overcome this edge enhancements or image deblurring is done. Deblurring is done using various methods like TV variational regularization models[3,4,5,6], Sparse representations[7,8],state of art dictionary learning methods[9,10],K-SVD[11],non local similarity and self similarity methods[12],iterative methods[13,14] and wavelet transforms[15,16,17].
The TV variational models[3,4,5,6] are the commonly used regularization models which favors the piecewise constant image structures which smoothens the fine details in the image. Sparse representations[7,8] have been used in various image processing applications along with the over complete dictionary techniques .Non local similarity measures[12] on the other hand preserves edge sharpness and preserves noise. Block matching techniques like BM3D algorithm along with the wiener filtering techniques results in efficient image restoration technique. Iterative methods and Wavelet transforms[15,16,17] like DCT, contourlets, ridgelets results in analytic form of dictionary learning which results in issues of the dynamic nature of medical images.

\subsection{Review of Deblurring Process:}

In this paper LPG PCA technique is used along with the aforementioned processes. The LPG PCA technique helps in grouping each and every pixel and its neighboring elements, so the learning process involves every object in the test images, which leads to the improvement in the efficiency of the process. In general, the energy of a signal will concentrate on a small subset of the PCA transformed dataset [2], while the energy of noise will evenly spread over the whole dataset. Therefore, by preserving only the most important subset of the transformed dataset and then conducting the inverse PCA transform, the noise could be significantly reduced while the signal being well recovered. Since the PCA [2] is applied to the noisy image without any data selection, noise residuals may be there in the image leading to the visual artifacts. So this is avoided by modeling a pixel and its nearest neighbor as the vector variable.

The training samples of this variable are selected by grouping the pixels with similar local spatial structures to the underlying one in the local window. With such an LPG procedure[2], the local statistics of the variables can be accurately computed so that the image edge structures can be well preserved after shrinkage in the PCA domain .LPG PCA is a spatially adaptive image representation technique so that it can better characterize the images.

By combining the LPG PCA algorithm with the sparse representations deblurring can be achieved in an efficient manner. The adaptive regularization is one of the sparse domain selection techniques which are used in the image deblurring process. In this the training datasets are used in specified adaptively regularized sub dictionaries and then used for the deblurring approaches. Since most of the images contain objects of similar form (points, 
lines, and curves) these dictionaries help in filling out the missing objects and removing the low frequency components. Such dictionaries can be learnt using the LPG PCA technique. The success of sparse representation owes to the development of $l 1$ norm optimization techniques [1], and the fact that the medical images are intrinsically sparse in some domain. The image restoration quality largely depends on whether the employed sparse domain can represent well the underlying image. Considering that the contents can vary significantly across different images or different patches in a single image, various sets of bases are learnt from a pre-collected dataset of example image patches, and then for a given patch to be processed, one set of bases are adaptively selected to characterize the local sparse domain.

In this paper the performance of the LPG PCA algorithm in deblurring process is analyzed in the CT and MRI images and performance of the same is said in terms of various image quality measurements.

The rest of the paper is structured as follows: Section2 reviews the mathematical procedure of PCA. The concepts of local pixel grouping are given in section3. Section4 describes the LPG PCA based deblurring algorithm, Performance analysis of various image quality measures are given in Section5.The results are discussed in section6 and Section7 concludes the paper.

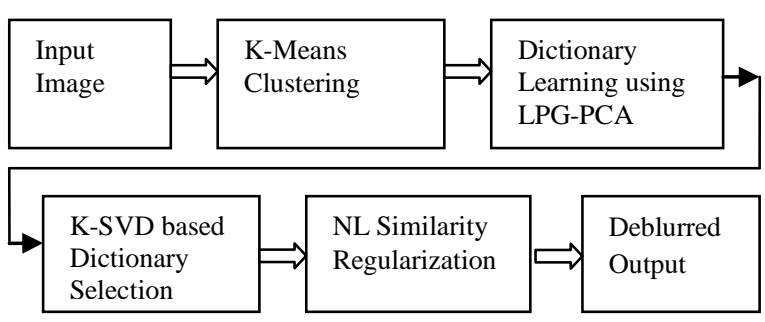

Fig1: Process of LPG PCA based deblurring.

\section{PRINCIPLE COMPONENT}

\section{ANALYSIS:}

Principle component analysis is a statistical decorrelation technique in which some basic steps are followed to perform the dictionary learning technique. First an input dataset is selected and the mean is subtracted from each of the data dimensions. The mean subtracted will be the average across each dimension. As a result a dataset with zero mean is produced. Then covariance matrix is calculated since a $2 \mathrm{D}$ image is taken as input. From the covariance matrix the eigen values and eigen vectors are calculated. The eigen values of the covariance matrix are the variances of the transformed data along the principal axes of the transformed space and eigen vectors are the principle components of the image. The principle components are chosen and a feature vector is formed. Based on the feature vector a new dataset is formed which will be devoid of information which is of less importance.

In order to explain the above mentioned concepts mathematically, let us consider $\mathrm{X}=\left[\mathrm{X}^{1} \mathrm{X}^{2} \ldots \mathrm{Xm}\right]^{\mathrm{T}}$ an $\mathrm{m}$ component vector variable and is denoted by

$$
\mathrm{X}=\left[\begin{array}{cccc}
\chi_{1}^{1} & \chi_{2}^{1} & \cdots & \chi_{1}^{n} \\
\vdots & \vdots & \vdots \\
\chi_{m}^{1} & \chi_{m}^{2} & \cdots & \chi_{m}^{n}
\end{array}\right]
$$

The sample matrix of $\chi$ where $\chi_{i}^{j}, \mathrm{j}=1,2 \ldots \mathrm{n}$ are discrete sample variables of $\chi_{i}, \mathrm{i}=1,2 \ldots \mathrm{m}$. The $\mathrm{ith}$ row of a sample matrix $\mathrm{X}$ is called the sample vector of $\chi_{i}$.

The mean value of $\chi_{i}$ is then calculated using the formula,

$$
\mu_{i}=\frac{1}{n} \sum_{j=1}^{n} \chi_{i}(j)
$$

And the sample vector $\chi_{i}$ is centralized as

$$
\overline{\chi_{i}}=\chi_{i}-\mu_{i}
$$

Then the covariance matrix of the centralized dataset is identified as,

$$
\Omega=\frac{1}{n} \overline{X X}^{\mathrm{T}}
$$

The goal of PCA is to find the orthonormal transformation matrix to decorrelate the covariance matrix. Due to the symmetrical property of covariance matrix it is written as,

$$
\Omega=\Phi \Lambda \Phi^{T}
$$

Where $\phi=\left[\phi_{1} \phi_{2} \ldots \phi_{m}\right]$ is a $m x m$ orthonormal eigen vector matrix and $\Lambda=\operatorname{diag}\left[\lambda_{1}, \lambda_{2}, . . \lambda_{m}\right]$ is the diagonal eigen value matrix. By setting the orthonormal transformation matrix, $\mathrm{P}$ to

$$
P=\Phi^{T}
$$

$\bar{X}$ can be decorrelated.

\section{LOCAL PIXEL GROUPING:}

Different grouping methods like block matching, $\mathrm{k}$ means clustering can be used to group the different training samples according to the training window in the central block. There are totally $(\mathrm{L}-\mathrm{K}+1)^{2}$ training blocks of $\chi_{n}$ in the $\mathrm{LxL}$ training window. The column samples vectors in the $\mathrm{KxK}$ window is represented by $\overrightarrow{\chi_{o}^{n}}$ and the sample vectors representing is represented by $\overrightarrow{\chi_{l}^{n}}$. The noiseless sample vectors of $\overrightarrow{\chi_{o}^{n}}$ and $\overrightarrow{\chi_{l}^{n}}$ can be easily calculated using,

$e_{i}=\frac{1}{m} \sum_{k=1}^{m} \overrightarrow{\chi_{o}^{n}}(k)-\overrightarrow{\chi_{l}^{n}}(k)^{2} \approx \frac{1}{m} \sum_{k=1}^{m} \overrightarrow{\chi_{o}}(k)-\overrightarrow{\chi_{l}}(k)^{2}+2 \sigma^{2}$

here the noise is white and uncorrelated. Since the medical images deal with random noise, noise removal is similar to those of the white noise. If

$$
e_{i}<T+2 \sigma^{2}
$$

Where $\mathrm{T}$ is a preset threshold, then $\overrightarrow{\chi_{l}^{n}}$ will be selected as the sample vector of $\chi_{n}$. The training datasets of $\chi_{n}$ is formed by, 


$$
\chi_{n}=\left[\overrightarrow{\chi_{o}^{n}} \overrightarrow{\chi_{1}^{n}} \ldots \overrightarrow{\chi_{n-1}^{n}}\right]
$$

The noiseless counterpart of $\chi_{n}$ is denoted by $\chi=$ $\left[\overrightarrow{\chi_{0}} \overrightarrow{\chi_{1}} \ldots \overrightarrow{\chi_{n-1}}\right]$.

After extracting the vector variables, the problem extends to the process of extracting the noiseless counterparts from noisy images. Once $\chi$ is estimated the central block and consequently the central underlying pixel can be extracted.

\section{LPG PCA DEBLURRING ALGORITHM:}

\subsection{Spatially adaptive Sparse Domain Selection:}

In this section sparse domain scheme which learns series of sub dictionaries and assigns adaptively each local patch a sub dictionary is explained. Let $x$ be an image vector, and $x_{i}=R_{i} x$, $i=1,2, \ldots, N$, be the $i^{\text {th }}$ patch (size: $\sqrt{n} \times \sqrt{n}$ ) vector of $x$, where $R_{i}$ is a matrix extracting patch $x_{i}$ from $x$. For patch $x_{i}$, suppose that a sub dictionary $\Phi_{k_{i}}$ is selected for it. Then, $x_{i}$ can be approximated as $\hat{x}_{i}=\Phi_{k_{i}} \alpha_{i},\left\|\alpha_{i}\right\|_{1} \leq T$, via sparse coding. The whole image $x$ can be reconstructed by averaging all the reconstructed patches $\hat{x}_{i}$, which can be mathematically written as,

$$
\hat{x}=\left(\sum_{i-1}^{N} R_{i}^{T} R_{i}\right)^{-1} \sum_{i-1}^{N}\left(R_{i}^{T} \Phi_{k_{i}} \alpha_{i}\right)
$$

One important procedure from the above scheme is the determination of $\Phi_{k_{i}}$ for each local patch. To facilitate the deblurring process, the sub-dictionaries $\left\{\Phi_{k}\right\}$ are learnt offline, and selected online from $\left\{\Phi_{k}\right\}$ the best fitted sub-dictionary to each patch $\boldsymbol{x}_{i}$.

\subsection{LPG PCA based Dictionary Learning:}

Cluster the dataset $S$ into $K$ clusters, and learn a sub-dictionary from each of the $K$ clusters. Apparently, the $K$ clusters are expected to represent the $K$ distinctive patterns in $S$. To generate perceptually meaningful clusters, the clustering is performed in a feature space. Let $S_{h}=\left[s_{1}{ }^{h}, s_{2}{ }^{h}, \ldots, s_{M}{ }^{h}\right]$ be the high-pass filtered dataset of $S$. The $K$-means algorithm is used to partition $S_{h}$ into $K$ clusters $\left\{C_{1}, C_{2}, \quad, C_{K}\right\}$ and denote by $\mu_{\mathrm{k}}$ the centroid of cluster $C_{k}$. Once $S_{h}$ is partitioned, the dataset $S$ can then be clustered into $K$ subsets $S_{k}, k=1,2, . ., K$, and $S_{k}$ is a matrix of dimension $n \times m_{k}$, where $m_{k}$ denotes the number of samples in $S_{k}$. Now the remaining problem is how to learn a sub-dictionary $\Phi_{k}$ from the cluster $S_{k}$ such that all the element in $S_{k}$ can be faithfully represented by $\Phi_{k}$ and this is solved by LPG PCA technique.

LPG PCA is applied to each sub-dataset $S_{k}$ to compute the principal components, from which the dictionary $\Phi_{k}$ is constructed. Denote by $\Omega_{k}$ the co-variance matrix of dataset $S_{k}$. By applying LPG PCA to $\Omega_{k}$, an orthogonal transformation matrix $P_{k}$ can be obtained. If $P_{k}$ is set as the dictionary and let $Z_{k}=P_{k}^{T} S_{k}$, then $\left\|S_{k}-P_{k} Z_{k}\right\|_{F}^{2}=\left\|S_{k}-P_{k} P_{k}^{T} S_{k}\right\|_{F}^{2}=0$. To make a better balance between the $l 1$-norm regularization term and $l 2$-norm approximation term in Eq. (10), the first $r$ most important eigenvectors are extracted in $P_{k}$ to form a dictionary $\Phi_{r}$, i.e. $\Phi_{r}=\left[p_{1}, p_{2}, \ldots p_{r}\right]$. Let $\Lambda_{r}=\Phi_{r}^{T} S_{k}$. Clearly, since not all the eigenvectors are used to form $\Phi_{r}$, the reconstruction error will increase with the decrease of $r$. However, the term $\left\|\Lambda_{r}\right\|_{1}$ will decrease. Therefore, the optimal value of $r$, denoted by $r o$, can be determined by,

$r_{o}=\arg \min _{r}\left\{\left\|S_{k}-\Phi_{r} \Lambda_{r}\right\|_{F}^{2}+\lambda\left\|\Lambda_{r}\right\|_{1}\right\}$

Finally, the sub-dictionary learned from sub-dataset $S_{k}$ is $\Phi_{k}=\left[p_{1}, p_{2}, \ldots p_{r}\right]$. Applying the above procedures to all the $K$ sub-datasets $S_{k}, K$ sub-dictionaries $\Phi_{k}$, can be obtained which will be used in the adaptive sparse domain selection process of each given image patch.

\subsection{K-SVD based Dictionary Selection:}

A sub dictionary $\Phi_{k}$ for each subset $S_{k}$ and centroids of $S_{k}$ are present. In the sparsity-based deblurring process, a subdictionary to each local patch of $x$ is assigned adaptively, spanning the adaptive sparse domain. Since the centroid $\mu_{k}$ of each cluster is available, and the best fitted sub-dictionary could be selected for $\hat{x}_{i}$ by comparing the high-pass filtered patch of $\hat{x}_{i}$, denoted by $\hat{x}_{i}^{h}$, to the centroid $\mu_{k}$. Let $U=\left[\mu_{1}, \mu_{2}, \ldots \ldots, \mu_{k}\right]$ be the matrix containing all the centroids. By applying SVD to the co-variance matrix of $U$, the PCA transformation matrix of $U$ can be obtained. Let $\Phi_{c}$ be the projection matrix composed by the first several most significant eigenvectors. The distance between $\hat{x}_{i}^{h}$ and $\mu_{k}$ in the subspace spanned by $\Phi_{c}$ is computed as,

$k_{i}=\arg \min _{r}\left\|\Phi_{c} \hat{x}_{i}^{h}-\Phi_{c} \mu_{k}\right\|_{2}$

By using Eq. (12), the $k_{i}^{t h}$ sub-dictionary $\Phi_{k_{i}}$ will be selected and assigned to patch $\hat{x}_{i}$. This process of deblurring is further improved by non local similarity constraints. With the fact that there are often many repetitive image structures in medical images, the image non-local redundancies can be very helpful in image enhancement.

\section{PERFORMANCE ANALYSIS:}

Performance analysis of LPG PCA algorithm in medical images are done by using various image quality measures like Peak Signal to Noise ratio (PSNR), Structural similarity index(SSIM),Average Difference(AD), Maximum Difference(MD) ,Mean Square Error (MSE), Normalized Absolute Error(NAE), Normalized Cross Correlation(NK), and Structural content(SC).These help in evaluating the visual quality of the image and the similarity between the intensity of the pixels. A good objective quality measure should well reflect the distortion on the image due to, for example, blurring, noise, compression, sensor inadequacy. The reason for their widespread choice is their mathematical tractability and it is often straightforward to design systems that minimize the MSE.

Raw error measures such as MSE may quantify the error in mathematical terms, and they are at their best with additive noise contamination, but they do not necessarily correspond to all aspects of the observer's visual perception of the errors, nor do 
they correctly reflect structural coding artifacts. For the performance analysis 20sets of images are taken in CT and MRI under various subject selections. The analysis measures are done by using sparse domain techniques with and without using LPG PCA algorithm. The PSNR values are found to be better in the LPG PCA based algorithm. Also it's obvious that local pixel grouping is effective from the change in PSNR values between the first and the second stage images.

The SNR is inversely proportional to spatial resolution, i.e., higher spatial resolution yields lower SNR. Thus, the spatial resolution of MR is limited by available SNR and the image acquisition time. Repeated acquisition and averaging could improve SNR in MRI; however, multiple averaging is limited by several factors such as limited breath-hold duration. An alternative approach to averaging is to use more SNR-efficient image acquisition techniques. For example, steady-state free precession MRI has superior SNR property compared to spoiled gradient echo sequences. So PSNR plays a major role in declaring the quality of the image. Also the structural similarity index (SSIM) is used to measure the visual perception of an image based on its similarity.

\section{RESULTS AND DISCUSSION:}

20 sets of CT and MRI images of size $(512 \times 512)$ are taken for performing the analysis. The results of CT and MRI images are shown below along with the performance analysis measures in the Table 1and2.As already discussed the deblurring process along with the LPG PCA algorithm will result in effective edge preservation.. The improvement of LPG PCA based deblurring process over general deblurring process is shown in Table 3 and 4 which shows the results of various image quality measures of CT and MRI images. Results of Table 3 are discussed in detail below.

It is found that LPG PCA based image restoration is effective in all the image quality measurements. The PSNR values have an average value of $36 \mathrm{~dB}$ in LPG PCA deblurring process compared to $33 \mathrm{~dB}$ in actual process, which shows the intensity difference between the two images. The improvement gain is $2.98 \mathrm{~dB}$ of PSNR value. The structural similarity measure shows average of 0.9744 compared to the 0.9547 and results in an average improvement of 0.019 . Lower the value of average difference higher the noise reduced which in turn shows the edge preservation characteristics. The average difference measures of LPG PCA based process results in 0.08 which shows the optimum difference of the deblurred image and the maximum difference results in the average improvement of -0.0636.This difference value shows the quality improvement of the image which in turn depicts the performance difference between the two processes. Lesser the mean square error value better the performance. The MSE value shows an average of -13.838 improvement.

The closeness between two images is measured in terms of correlation function. The normalized cross correlation shows the similarity between the two images and hence they are complimentary to difference based measures. Normalized absolute error shows how far the deblurred image is related with the original image. Higher the value of NAE, lesser the image quality. Here the results shows that NAE is about -0.0161 , which shows the improvement of image quality in LPG PCA based process. And finally structural content (SC) shows the similarity of two images.SC value improvement is found to be 0.0029 .

The above mentioned image quality measures are also measured for MRI images.Table4 depicts the improvement ratio in MRI images and results are found to be $5.1375 \mathrm{~dB}$ (PSNR), 0.0475 (SSIM), 0.0391 (AD), -14.2685 (MD), -33.9411 (MSE), 0.05115 (NAE), -0.05021 (NK), 0.0065 (SC). These results shows that LPG PCA based deblurring algorithm results in improved image quality of both the CT and MRI images.
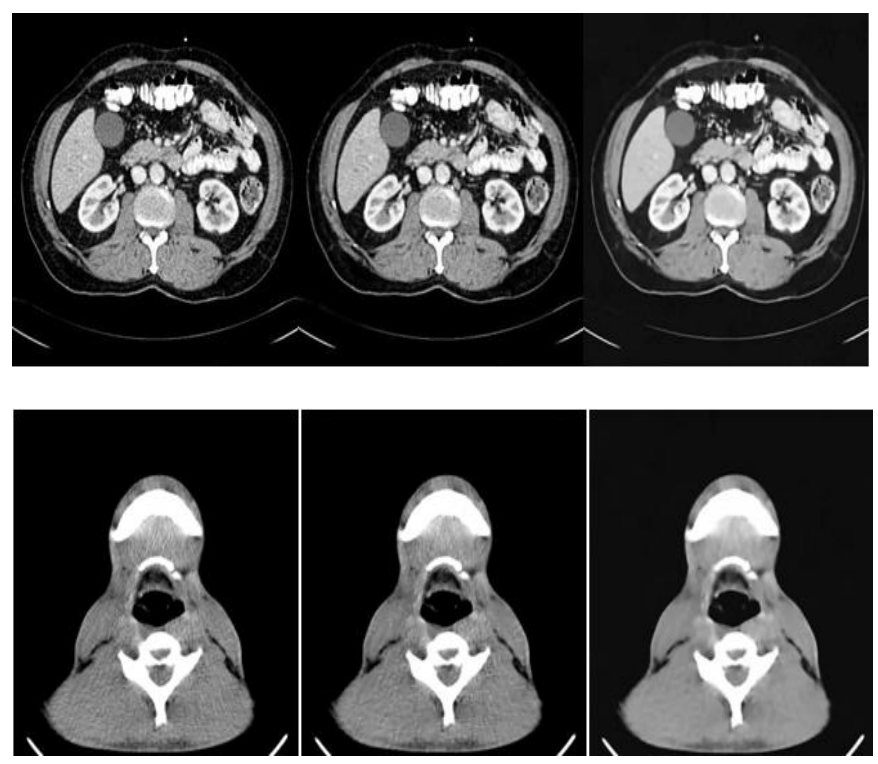

Fig2: Deblurring Results of CT Image Using LPG PCA Algorithm (Input Image, Deblurred Image without LPG PCA, Deblurred Image With LPG PCA)
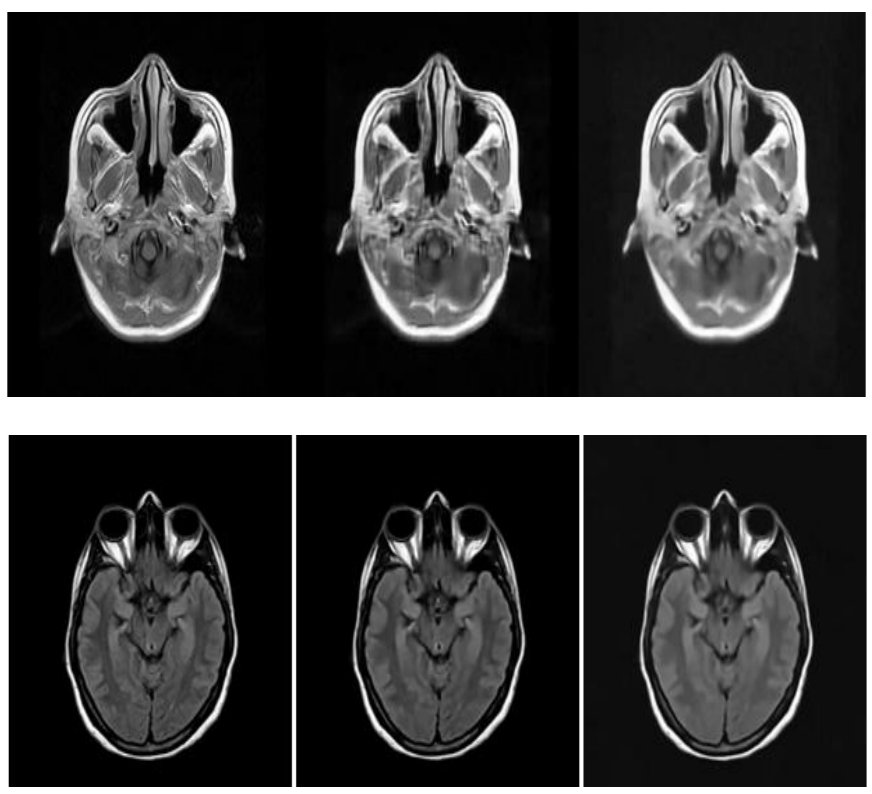

Fig3: Deblurring Results of MRI Image Using LPG PCA Algorithm (Input Image, Deblurred Image without LPG PCA, Deblurred Image with LPG PCA) 
Table1: Deblurring Results of CT images

\begin{tabular}{|c|c|c|c|c|c|c|c|c|c|c|c|c|c|c|c|c|}
\hline \multirow{2}{*}{ Image } & \multicolumn{8}{|c|}{ DEBLURRING WITHOUT LPG-PCA } & \multicolumn{8}{|c|}{ DEBLURRING WITH LPG-PCA } \\
\hline & PSNR & SSIM & $\mathrm{AD}$ & MD & MSE & NAE & NK & SC & PSNR & SSIM & $\mathrm{AD}$ & MD & MSE & NAE & NK & SC \\
\hline 1 & 29.75 & 0.87 & 0.019 & 103.92 & 68.95 & 0.09 & 1.001 & 0.99 & 34.54 & 0.95 & 0.073 & 103.10 & 22.85 & 0.04 & 1.000 & 1.00 \\
\hline 2 & 33.02 & 0.95 & 0.294 & 122.46 & 32.46 & 0.04 & 1.001 & 0.99 & 35.55 & 0.97 & 0.096 & 102.81 & 18.13 & 0.03 & 1.001 & 1.00 \\
\hline 3 & 34.24 & 0.93 & 0.246 & 88.25 & 24.49 & 0.04 & 1.001 & 1.01 & 38.05 & 0.96 & 0.080 & 53.08 & 10.20 & 0.02 & 1.000 & 1.00 \\
\hline 4 & 35.23 & 0.92 & 0.28 & 55.91 & 19.51 & 0.04 & 1.004 & 1.01 & 40.34 & 0.97 & 0.086 & 58.78 & 6.01 & 0.02 & 1.000 & 1.00 \\
\hline 5 & 34.49 & 0.98 & 0.071 & 91.75 & 23.11 & 0.03 & 1.001 & 1.05 & 32.68 & 0.97 & 0.252 & 100.57 & 35.09 & 0.03 & 1.001 & 0.99 \\
\hline 6 & 33.71 & 0.94 & -0.016 & 136.24 & 27.68 & 0.04 & 1.001 & 1.02 & 38.02 & 0.96 & 0.080 & 58.93 & 10.26 & 0.02 & 1.001 & 1.00 \\
\hline 7 & 32.4 & 0.95 & -0.048 & 166.99 & 37.4 & 0.03 & 1.001 & 0.99 & 36.2 & 0.97 & 0.023 & 63.03 & 15.62 & 0.02 & 1.001 & 1.00 \\
\hline 8 & 34.73 & 0.93 & -0.009 & 75.55 & 21.87 & 0.04 & 1.001 & 0.99 & 40.34 & 0.97 & 0.089 & 45.65 & 6.01 & 0.02 & 1.000 & 1.00 \\
\hline 9 & 31.21 & 0.88 & -0.019 & 175.85 & 49.19 & 0.05 & 1.001 & 0.99 & 36.39 & 0.92 & 0.044 & 61.87 & 14.93 & 0.02 & 1.001 & 1.00 \\
\hline 10 & 33.85 & 0.94 & -0.017 & 76.91 & 26.82 & 0.04 & 1.001 & 1.01 & 37.97 & 0.97 & 0.073 & 57.04 & 10.39 & 0.02 & 1.001 & 1.00 \\
\hline 11 & 36.12 & 0.96 & -0.049 & 110.25 & 15.9 & 0.02 & 1.002 & 0.99 & 37.43 & 0.98 & 0.077 & 74.27 & 11.74 & 0.01 & 1.001 & 1.00 \\
\hline 12 & 31.9 & 0.98 & -0.028 & 191.72 & 41.95 & 0.03 & 1.003 & 0.99 & 34.59 & 0.98 & 0.070 & 92.27 & 22.58 & 0.02 & 1.001 & 1.00 \\
\hline 13 & 34.84 & 0.93 & -0.067 & 107.24 & 21.32 & 0.03 & 1.002 & 0.99 & 37.21 & 0.98 & 0.075 & 74.66 & 12.37 & 0.02 & 1.001 & 1.00 \\
\hline 14 & 32.84 & 0.98 & 0.004 & 181.94 & 33.79 & 0.04 & 1.002 & 0.99 & 34.45 & 0.98 & 0.094 & 80.57 & 23.32 & 0.03 & 1.001 & 1.00 \\
\hline 15 & 33.12 & 0.96 & -0.039 & 178.63 & 31.72 & 0.03 & 1.002 & 0.99 & 35.71 & 0.98 & 0.076 & 69.68 & 17.44 & 0.02 & 1.001 & 1.00 \\
\hline 16 & 34.8 & 0.96 & 0.042 & 99.31 & 21.51 & 0.05 & 1.002 & 0.99 & 36.58 & 0.98 & 0.097 & 78.45 & 14.29 & 0.03 & 1.001 & 1.00 \\
\hline 17 & 34.82 & 0.96 & 0.047 & 80.51 & 21.43 & 0.05 & 1.002 & 0.99 & 36.28 & 0.98 & 0.098 & 68.44 & 15.31 & 0.03 & 1.001 & 1.00 \\
\hline 18 & 35.04 & 0.96 & 0.045 & 76.69 & 20.37 & 0.05 & 1.002 & 0.99 & 36.72 & 0.98 & 0.100 & 76.82 & 13.83 & 0.03 & 1.001 & 1.00 \\
\hline 19 & 34.59 & 0.96 & 0.039 & 75.64 & 22.61 & 0.05 & 1.002 & 0.99 & 36.38 & 0.98 & 0.095 & 77.57 & 14.96 & 0.03 & 1.001 & 1.00 \\
\hline 20 & 34.18 & 0.95 & 0.054 & 82.64 & 24.86 & 0.05 & 1.002 & 0.99 & 36.42 & 0.98 & 0.098 & 63.90 & 14.84 & 0.03 & 1.001 & 1.00 \\
\hline
\end{tabular}

Table2: Deblurring Results of MRI images

\begin{tabular}{|c|c|c|c|c|c|c|c|c|c|c|c|c|c|c|c|c|}
\hline \multirow[b]{2}{*}{ Image } & \multicolumn{8}{|c|}{ DEBLURRING WITHOUT LPG-PCA } & \multicolumn{8}{|c|}{ DEBLURRING WITH LPG-PCA } \\
\hline & PSNR & SSIM & AD & MD & MSE & NAE & NK & SC & PSNR & SSIM & AD & MD & MSE & NAE & NK & SC \\
\hline 1 & 29.28 & 0.90 & 0.063 & 89.95 & 76.73 & 0.07 & 1.001 & 0.99 & 30.96 & 0.92 & 0.027 & 75.17 & 52.13 & 0.05 & 1.001 & 0.99 \\
\hline 2 & 33.6 & 0.90 & 0.152 & 56.78 & 28.42 & 0.05 & 1.000 & 0.99 & 37.52 & 0.97 & 0.055 & 46.06 & 11.50 & 0.02 & 1.000 & 1.00 \\
\hline 3 & 33.23 & 0.93 & 0.054 & 74.26 & 30.93 & 0.05 & 1.001 & 0.99 & 35.95 & 0.96 & 0.009 & 64.53 & 16.54 & 0.03 & 1.001 & 1.00 \\
\hline 4 & 28.8 & 0.89 & 0.172 & 93.52 & 85.69 & 0.11 & 1.003 & 0.97 & 30.29 & 0.93 & 0.070 & 95.79 & 60.85 & 0.07 & 1.002 & 0.98 \\
\hline 5 & 31.45 & 0.90 & 0.158 & 92.76 & 46.59 & 0.07 & 1.001 & 0.99 & 34.62 & 0.95 & 0.068 & 73.92 & 22.43 & 0.04 & 1.000 & 1.00 \\
\hline 6 & 29.51 & 0.92 & -0.074 & 95.25 & 72.80 & 0.10 & 1.001 & 0.98 & 36.02 & 0.97 & 0.009 & 66.03 & 16.27 & 0.03 & 1.000 & 1.00 \\
\hline 7 & 30.53 & 0.92 & -0.088 & 92.56 & 57.54 & 0.09 & 1.001 & 0.99 & 37.35 & 0.97 & 0.012 & 70.29 & 11.96 & 0.03 & 1.000 & 1.00 \\
\hline 8 & 29.71 & 0.81 & -0.065 & 75.93 & 69.46 & 0.10 & 1.001 & 0.99 & 38.87 & 0.98 & 0.119 & 57.79 & 8.44 & 0.02 & 1.000 & 1.00 \\
\hline 9 & 38.23 & 0.95 & -0.010 & 38.67 & 9.76 & 0.05 & 1.000 & 1.00 & 42.48 & 0.98 & 0.141 & 29.03 & 3.68 & 0.02 & 1.000 & 1.00 \\
\hline 10 & 28.77 & 0.79 & -0.055 & 86.28 & 86.27 & 0.11 & 1.000 & 0.99 & 37.98 & 0.97 & 0.053 & 45.09 & 10.36 & 0.02 & 1.000 & 1.00 \\
\hline 11 & 32.11 & 0.82 & -0.003 & 61.97 & 40.02 & 0.10 & 0.999 & 0.99 & 39.6 & 0.98 & -0.027 & 37.79 & 7.12 & 0.02 & 1.000 & 1.00 \\
\hline 12 & 31.2 & 0.90 & -0.035 & 63.79 & 49.28 & 0.12 & 1.001 & 0.99 & 30.4 & 0.94 & 0.071 & 95.64 & 59.31 & 0.07 & 1.002 & 0.99 \\
\hline 13 & 30.48 & 0.92 & -0.032 & 70.63 & 58.17 & 0.10 & 1.002 & 0.98 & 36.16 & 0.97 & 0.074 & 67.68 & 15.73 & 0.03 & 1.000 & 1.00 \\
\hline 14 & 36.31 & 0.95 & -0.028 & 34.23 & 15.21 & 0.05 & 1.000 & 1.00 & 41.69 & 0.98 & 0.091 & 30.98 & 4.41 & 0.02 & 1.000 & 1.00 \\
\hline 15 & 29.11 & 0.89 & -0.017 & 101.22 & 79.73 & 0.11 & 1.001 & 0.98 & 34.61 & 0.95 & 0.069 & 74.16 & 22.51 & 0.04 & 1.000 & 1.00 \\
\hline 16 & 27.3 & 0.81 & 0.040 & 76.66 & 121.16 & 0.10 & 1.000 & 0.99 & 32.45 & 0.89 & 0.044 & 53.34 & 37.00 & 0.04 & 1.000 & 1.00 \\
\hline 17 & 37.76 & 0.97 & -0.029 & 51.80 & 10.90 & 0.06 & 1.001 & 0.99 & 40.81 & 0.98 & 0.107 & 33.60 & 5.40 & 0.02 & 1.000 & 1.00 \\
\hline 18 & 31.54 & 0.84 & 0.000 & 51.15 & 45.60 & 0.08 & 1.000 & 0.99 & 37.55 & 0.95 & -0.007 & 36.71 & 11.44 & 0.03 & 1.001 & 1.00 \\
\hline 19 & 28.74 & 0.83 & 0.030 & 65.92 & 86.87 & 0.09 & 1.000 & 0.99 & 34.3 & 0.93 & 0.031 & 48.22 & 24.13 & 0.03 & 1.000 & 1.00 \\
\hline 20 & 35.23 & 0.96 & -0.014 & 42.56 & 19.51 & 0.06 & 1.000 & 0.99 & 40.51 & 0.99 & -0.012 & 28.69 & 10.60 & 0.03 & 0.001 & 1.00 \\
\hline
\end{tabular}

Table3: Improvement ratio of CT images

\begin{tabular}{|l|c|c|c|c|c|c|c|}
\hline \multicolumn{7}{|c|}{ IMPROVEMENT RATIO of CT IMAGES } \\
\hline PSNR & SSIM & AD & MD & MSE & NAE & NK & SC \\
\hline 4.74 & 0.02 & 0.05 & -0.04 & -46.11 & -0.05 & 0.000 & 0.006 \\
5.07 & 0.01 & 0.07 & -0.06 & -14.33 & -0.02 & 0.000 & 0.002 \\
3.81 & 0.01 & 0.06 & -0.04 & -14.30 & -0.02 & 0.000 & 0.002 \\
5.11 & 0.03 & 0.06 & -0.03 & -13.50 & -0.02 & 0.000 & 0.001 \\
1.81 & 0.01 & 0.18 & -0.18 & 11.98 & 0.01 & 0.000 & -0.001 \\
4.31 & 0.02 & 0.10 & -0.07 & -17.42 & -0.02 & 0.000 & 0.002 \\
3.80 & 0.04 & 0.07 & -0.04 & -21.79 & -0.01 & 0.000 & 0.001 \\
2.19 & 0.01 & 0.10 & -0.09 & -15.85 & -0.02 & 0.000 & 0.002 \\
5.18 & 0.02 & 0.06 & -0.04 & -34.27 & -0.02 & 0.000 & 0.002 \\
4.12 & 0.03 & 0.09 & -0.06 & -16.43 & -0.02 & 0.000 & 0.002 \\
1.31 & 0.03 & 0.13 & -0.09 & -4.16 & -0.01 & -0.001 & 0.003 \\
2.69 & 0.04 & 0.10 & -0.06 & -19.37 & -0.01 & -0.001 & 0.004 \\
2.37 & 0.03 & 0.14 & -0.12 & -8.95 & -0.01 & -0.002 & 0.004 \\
1.61 & 0.00 & 0.09 & -0.09 & -10.46 & -0.01 & -0.002 & 0.005 \\
2.59 & 0.02 & 0.11 & -0.09 & -14.27 & -0.01 & -0.001 & 0.004 \\
1.76 & 0.01 & 0.05 & -0.04 & -7.22 & -0.02 & -0.001 & 0.004 \\
1.46 & 0.01 & 0.05 & -0.04 & -6.12 & -0.02 & -0.001 & 0.004 \\
1.68 & 0.02 & 0.06 & -0.04 & -6.54 & -0.02 & -0.001 & 0.004 \\
1.79 & 0.02 & 0.06 & -0.04 & -7.65 & -0.02 & -0.001 & 0.004 \\
2.24 & 0.02 & 0.04 & -0.02 & -10.01 & -0.02 & -0.001 & 0.004 \\
\hline \multicolumn{70}{|c|}{}
\end{tabular}

Table4: Improvement Ratio of MRI Image

\begin{tabular}{|r|r|r|r|r|r|r|r|}
\hline \multicolumn{7}{|c|}{ IMPROVEMENT RATIO of MRI IMAGES } \\
\hline PSNR & SSIM & AD & \multicolumn{1}{|c|}{ MD } & \multicolumn{1}{l|}{ MSE } & NAE & \multicolumn{1}{l|}{ NK } & \multicolumn{1}{l|}{ SC } \\
\hline 1.68 & 0.03 & -0.04 & -14.78 & -24.60 & -0.02 & -0.001 & 0.005 \\
3.92 & 0.06 & -0.10 & -10.72 & -16.92 & -0.03 & 0.000 & 0.004 \\
2.72 & 0.04 & -0.05 & -9.73 & -14.39 & -0.02 & 0.000 & 0.004 \\
1.49 & 0.07 & -0.10 & 2.27 & -24.84 & -0.04 & -0.001 & 0.011 \\
3.17 & 0.07 & -0.09 & -18.84 & -24.16 & -0.03 & -0.001 & 0.006 \\
6.51 & 0.08 & 0.08 & -29.22 & -56.53 & -0.07 & -0.001 & 0.012 \\
6.82 & 0.09 & 0.10 & -22.27 & -45.58 & -0.07 & -0.001 & 0.010 \\
9.16 & 0.04 & 0.18 & -18.14 & -61.02 & -0.08 & -0.001 & 0.012 \\
4.25 & 0.02 & 0.15 & -9.64 & -6.09 & -0.03 & -0.001 & 0.002 \\
9.21 & 0.08 & 0.11 & -41.19 & -75.91 & -0.09 & 0.000 & 0.012 \\
8.50 & 0.06 & -0.02 & -24.17 & -32.90 & -0.08 & 0.001 & 0.006 \\
6.32 & 0.03 & 0.11 & 31.85 & 10.03 & -0.05 & 0.002 & -0.002 \\
5.68 & 0.04 & 0.11 & -2.95 & -42.44 & -0.07 & -0.001 & 0.013 \\
5.38 & 0.03 & 0.12 & -3.25 & -10.80 & -0.03 & -0.001 & 0.004 \\
5.50 & 0.06 & 0.09 & -27.06 & -57.22 & -0.07 & -0.001 & 0.012 \\
5.15 & 0.08 & 0.00 & -23.32 & -84.15 & -0.06 & 0.001 & 0.007 \\
3.05 & 0.02 & 0.14 & -18.20 & -5.51 & -0.03 & -0.001 & 0.004 \\
6.01 & 0.02 & -0.01 & -14.45 & -34.16 & -0.06 & 0.001 & 0.004 \\
5.56 & 0.01 & 0.00 & -17.70 & -62.73 & -0.05 & 0.001 & 0.004 \\
2.67 & 0.02 & 0.00 & -13.87 & -8.91 & -0.03 & -1.000 & 0.002 \\
\hline
\end{tabular}




\section{CONCLUSION:}

This paper presented a detailed Performance analysis of local pixel grouping based principal component analysis algorithm in medical images using various image quality measures and results are discussed. PCA is a statistical decorrelation technique which is already used in many pattern recognition and signal processing applications. Image local structures are preserved by modeling the local pixel and its nearest neighbors as a vector variable. PCA transformation matrix was calculated for the vector variable and adaptively trained for local window of the image. Deblurring is done using adaptive regularization along with LPG PCA algorithm for dictionary learning. This was analyzed using various image quality measures and results were discussed. Results show that there is a noticeable improvement in LPG PCA based deblurring process, which results in the improvement of the image quality without degradation of local structures.

\section{REFERENCES}

[1] A. Buades, B. Coll, and J. M. Morel, "A review of image denoising algorithms, with a new one," Multisc. Model. Simulat., vol. 4, no. 2, pp. 490.530, 2005.

[2] Lei Zhang, "PCA-Based Spatially Adaptive Denoising of CFA Images for Single-Sensor Digital Cameras", IEEE Transactions on Image processing, vol. 18, no. 4, april 2009.

[3] M.Welk, D.Theis, J.Weickert, "Variational deburring of images with uncertain and spatially variant blurs. Pattern recognition," Mathematical image analysis group, Sarland university, Germany, vol.8, pp.33-40, 2005.

[4] T. Chan, S. Esedoglu, F. Park, and A. Yip, "Recent developments in total variation image restoration,"Mathematical Models of Computer Vision, N. Paragios, Y. Chen, and O. Faugeras, Eds. New York:Springer Verlag, 2005.

[5] J. Oliveira, J. M. Bioucas-Dia, M. Figueiredo, and, "Adaptive total variation image deblurring: a majorization-minimization approach," Signal Processing, vol. 89, no. 9, pp. 1683-1693, Sep. 2009.

[6] A. Beck and M. Teboulle, "Fast gradient-based algorithms for constrained total variation image

denoising and deblurring problems," IEEE Trans. On Image Process., vol. 18, no. 11, pp. 2419-2434,Nov. 2009 .
[7] Weisheng Donga,b, Lei Zhangb, "Image deblurring and Super-resolution by Adaptive Sparse Domain Selection and Adaptive Regularization", IEEE Transactions on Image Processing, vol. 20, no. 7, pp. 1838-1857, 2011.

[8] K. Dabov, A. Foi, V. Katkovnik, and K. Egiazarian, "Image restoration by sparse 3D transform-domain collaborative filtering," in Society of Photo-Optical Instrumentation Engineers (SPIE) Conference Series,vol. 6812,2008

[9] R. Rubinstein, M. Zibulevsky, and M. Elad, "Double sparsity: Learning Sparse Dictionaries for Sparse Signal Approximation," IEEE Trans. Signal Processing, vol. 58, no. 3, pp. 1553-1564, March 2010.

[10] R. Rubinstein, A.M. Bruckstein, and M. Elad, "Dictionaries for sparse representation modeling,"Proceedings of IEEE, Special Issue on Applications of Compressive Sensing \& Sparse Representation,vol. 98, no. 6, pp. 1045-1057, June, 2010.

[11] M. Aharon, M. Elad, and A. Bruckstein, "K-SVD: an algorithm for designing overcomplete dictionaries for sparse representation," IEEE Trans. Signal Process., vol. 54, no. 11, pp. 4311-4322, Nov. 2006.

[12] S. Kindermann, S. Osher, and P. W. Jones, "Deblurring and denoising of images by nonlocal functionals," Multiscale Modeling and Simulation, vol. 4, no. 4, pp. 1091-1115, 2005.

[13] Biemond, J. ,Lagendijk, R.L., Mersereau, R.M., "Iterative methods for image deblurring",Proceedings of IEEE,volume78,issue5,pg:856-883,May1990.

[14] Ge Wang,Snyder, D.L.,O'Sullivan, J.A., Vannier, M.W., "Iterative deblurring for CT Metal artifact reduction",IEEE Transactions on medical imaging,volume15,issue5,pg:657-664,1996.

[15] Vikas D Patil, Sachin D. Ruikar, "PCA Based Image Enhancement in Wavelet Domain", International Journa of Engineering Trends and Technology- Volume3Issue12012.

[16] Khare, A.,Shanker Tiwary, U., "A New Method for Deblurring and Denoising of Medical Images using Complex Wavelet Transform",IEEE Transactions on engineering in medicine and biology society,pg:18971900,2006

[17] Hui Ji and Kang Wang, "Robust Image Deblurring With an Inaccurate Blur Kernel", IEEE Transactions on Image processing, vol. 21, no. 4, april 2012. 